\title{
Active noise cancellation in a suspended interferometer
}

Jennifer C. Driggers, Matthew Evans, Keenan Pepper, and Rana Adhikari

Citation: Review of Scientific Instruments 83, 024501 (2012); doi: 10.1063/1.3675891

View online: https://doi.org/10.1063/1.3675891

View Table of Contents: http://aip.scitation.org/toc/rsi/83/2

Published by the American Institute of Physics

\section{Articles you may be interested in}

State observers and Kalman filtering for high performance vibration isolation systems

Review of Scientific Instruments 85, 034501 (2014); 10.1063/1.4866659

The advanced LIGO input optics

Review of Scientific Instruments 87, 014502 (2016); 10.1063/1.4936974

Design of a tuned mass damper for high quality factor suspension modes in Advanced LIGO

Review of Scientific Instruments 88, 035117 (2017); 10.1063/1.4978796

A programmable broadband low frequency active vibration isolation system for atom interferometry Review of Scientific Instruments 85, 093109 (2014); 10.1063/1.4895911

Thermal effects in the Input Optics of the Enhanced Laser Interferometer Gravitational-Wave Observatory interferometers

Review of Scientific Instruments 83, 033109 (2012); 10.1063/1.3695405

Small optic suspensions for Advanced LIGO input optics and other precision optical experiments

Review of Scientific Instruments 87, 114504 (2016); 10.1063/1.4967716

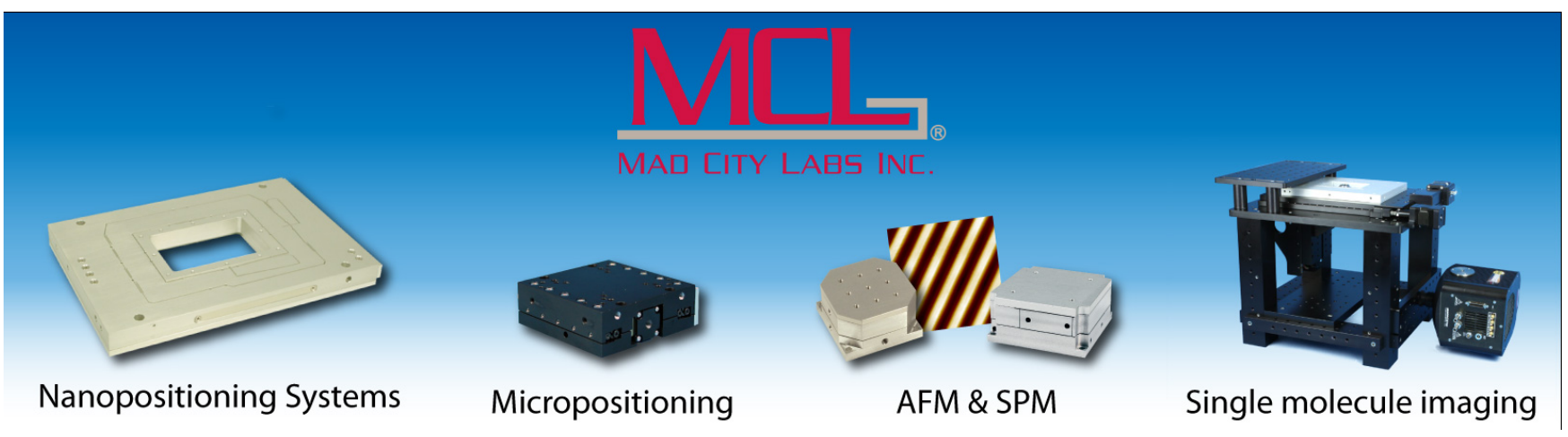




\title{
Active noise cancellation in a suspended interferometer
}

\author{
Jennifer C. Driggers, ${ }^{1, a)}$ Matthew Evans, ${ }^{2}$ Keenan Pepper, ${ }^{3}$ and Rana Adhikari ${ }^{1}$ \\ ${ }^{1}$ LIGO Laboratory, California Institute of Technology, Pasadena, California 91125, USA \\ ${ }^{2}$ LIGO Laboratory, Massachusetts Institute of Technology, Cambridge, Massachusetts 02139, USA \\ ${ }^{3}$ Department of Physics, University of California, Berkeley, California 94720, USA
}

(Received 24 June 2011; accepted 15 December 2011; published online 2 February 2012)

\begin{abstract}
We demonstrate feed-forward vibration isolation on a suspended Fabry-Perot interferometer using Wiener filtering and a variant of the common least mean square adaptive filter algorithm. We compare the experimental results with theoretical estimates of the cancellation efficiency. Using data from the recent Laser Interferometer Gravitational Wave Observatory (LIGO) Science Run, we also estimate the impact of this technique on full scale gravitational wave interferometers. In the future, we expect to use this technique also to remove acoustic, magnetic, and gravitational noise perturbations from the LIGO interferometers. This noise cancellation technique is simple enough to implement in standard laboratory environments and can be used to improve signal-to-noise ratio for a variety of high precision experiments. (C) 2012 American Institute of Physics. [doi:10.1063/1.3675891]
\end{abstract}

\section{INTRODUCTION}

The next generation of interferometers for gravitationalwave detection, including the Laser Interferometer Gravitational Wave Observatory (LIGO), will have unprecedented sensitivity to astrophysical events. ${ }^{1}$ At low frequencies $(\sim 10 \mathrm{~Hz})$ it is likely that the displacement noise of the suspended mirrors will be limited at the $10^{-20} \mathrm{~m} / \sqrt{\mathrm{Hz}}$ level by fluctuations in the Newtonian gravitational forces. ${ }^{2-4}$ The sources of the fluctuations are density perturbations in the environment (e.g., seismic and acoustic) and mechanical vibrations of the nearby experimental apparatus. While care will be taken to mitigate the sources of all of these fluctuations, further reductions of this Newtonian noise may be made by carefully measuring the source terms and subtracting them from the data stream (offline) or in hardware by applying cancellation forces to the mirror.

To demonstrate the efficacy of this technique, we demonstrate below that offline subtraction of seismic noise can be done using static Wiener filtering based on an array of seismic sensors. The online adaptive subtraction is also shown to approach the "optimal" Wiener limit. ${ }^{5}$ Through nonlinear processes, seismic noise below $10 \mathrm{~Hz}$ has been shown to limit the performance of gravitational-wave detectors. This technique will prove to be of substantial value in reducing the non-stationarity of the detectors.

\section{STATIC WIENER FILTERING}

To find a linear filter that will improve a chosen signal, we must first define what it means to "improve" the signal. The figure of merit $(\xi)$ that we use for calculating the Wiener filters in this case is the expectation value of the square of the error signal $(\vec{e})$, where the error signal is defined as the difference between the target signal to be minimized and the estimate of that signal calculated from the filtered witness

\footnotetext{
a)Electronic mail: jenne@ caltech.edu.
}

channels.

$$
\xi \equiv E\left[e^{2}(n)\right]=E\left[d^{2}(n)\right]-2 \vec{w}^{\mathrm{T}} p+\vec{w}^{\mathrm{T}} R \vec{w} .
$$

Here, $E[*]$ indicates the expectation value of $*, \vec{w}$ represents the tap weights of the filter, $d(n)$ is the target signal which we would like to minimize, $\vec{p}$ is the cross-correlation vector between the witness and target signals, and $R$ is the autocorrelation matrix for the witness channels. When we solve Eq. (1) by setting

$$
\frac{d \xi}{d w_{i}}=0
$$

we find

$$
R \vec{w}_{\text {optimum }}=\vec{p} .
$$

Equation (3) finds the finite impulse response (FIR) filter coefficients which minimize the rms of the error $\vec{e}$ by optimizing the estimate of the transfer function between the witness sensors and the target signal. Since the matrix $R$ is Block Toeplitz, we take advantage of the Levinson-Durbin ${ }^{6}$ method of solving problems of the form $\vec{b}=M \vec{a}$, where $M$ is a Toeplitz matrix. The Levinson method is considered weakly stable, as it is susceptible to numerical round-off errors when the matrix is close to degenerate (i.e., two or more witness sensors carry nearly identical information about the noise source). For well conditioned matrices it is much faster than brute force inversion of the matrix. ${ }^{7}$

The filtered output of the witness signals is $y(n)=\vec{w}^{\mathrm{T}} \vec{x}$ and $e(n)=d(n)-y(n)=d(n)-\vec{w}^{\mathrm{T}} \vec{x}$ is the filtered or minimized target signal, where $\vec{x}$ is the independent witness signal measuring our noise source. This new minimized signal $\vec{e}$ represents what the disturbance $\vec{d}$ would be if we were able to subtract the seismic noise before it entered the system.

Since we are using this feed-forward technique to reduce noise in the length of our cavities, and the cavity length carries gravitational wave information, we must be careful not to subtract the science signal along with the noise. For seismic noise, we are trying to cancel noise below $10 \mathrm{~Hz}$, while the gravitational wave signal band is above $10 \mathrm{~Hz}$. Even so, if 


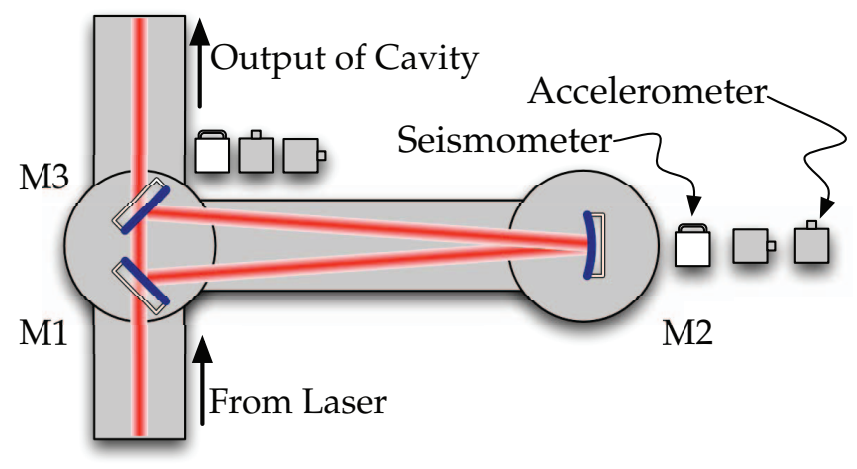

FIG. 1. (Color online) Locations of seismometers and accelerometers in relation to the cavity mirrors. Round trip length of the triangular cavity is $27 \mathrm{~m}$.

we use a filter of the same length as the data stream, we could perfectly cancel everything, including seismic noise and gravitational wave information. However, given the short length of our filters and the long stretches of data we calculate the subtraction over, the distortion of the gravitational wave signals should not be significant.

While the Wiener filtering occurs entirely in the timedomain, we examine plots in the frequency-domain of the filtered and unfiltered signals to determine the level of subtraction achieved. In Sec. III, we describe the Wiener simulations on a cavity in our lab. In Sec. IV, we make similar estimates for one of the $4 \mathrm{~km}$ LIGO interferometers. Finally, in Sec. V we demonstrate the performance of a real-time seismic noise cancellation system in the lab.

\section{WIENER FILTERING AT THE 40 m INTERFEROMETER}

At our $40 \mathrm{~m}$ prototype interferometer ${ }^{8}$ lab at Caltech, both static Wiener filtering and adaptive filtering algorithms have been applied to a suspended Fabry-Perot triangular ring cavity's feedback signal. We have used 2 Güralp CMG-40T seismometers and several Wilcoxon 731A accelerometers as our independent witness channels ( $\vec{x}$ in Sec. II), and the lowfrequency feedback signal for the cavity length as the target channel ( $\vec{d}$ in Eq. (1)) to reduce.

Figure 1 shows the locations of the witness sensors relative to the cavity mirrors. The mirrors of the cavity are suspended as pendulums with a resonance of $\sim 1 \mathrm{~Hz}$ to mechanically filter high frequency noise, with the suspensions sitting on vibration isolation stacks to further isolate the optics from ground motion. The "stacks" are a set of 3 legs supporting the optical table on which the mirror sits, with each of the legs consisting of alternating layers of stainless steel masses and elastomer springs. ${ }^{9,10}$

We used MATLAB to import the data for the length feedback signal for our cavity, and to construct and apply the Wiener filters. Since the feedback control bandwidth is $\simeq 50 \mathrm{~Hz}$, the feedback signal can be used as an accurate measure of the seismic disturbance at low frequencies. In Figure 2, we show results of a day-long simulation study. This study was done to determine the length of time we can use a set of static filters before updating. We use $1 \mathrm{~h}$ of data to train and calculate a single Wiener filter, and then apply that filter
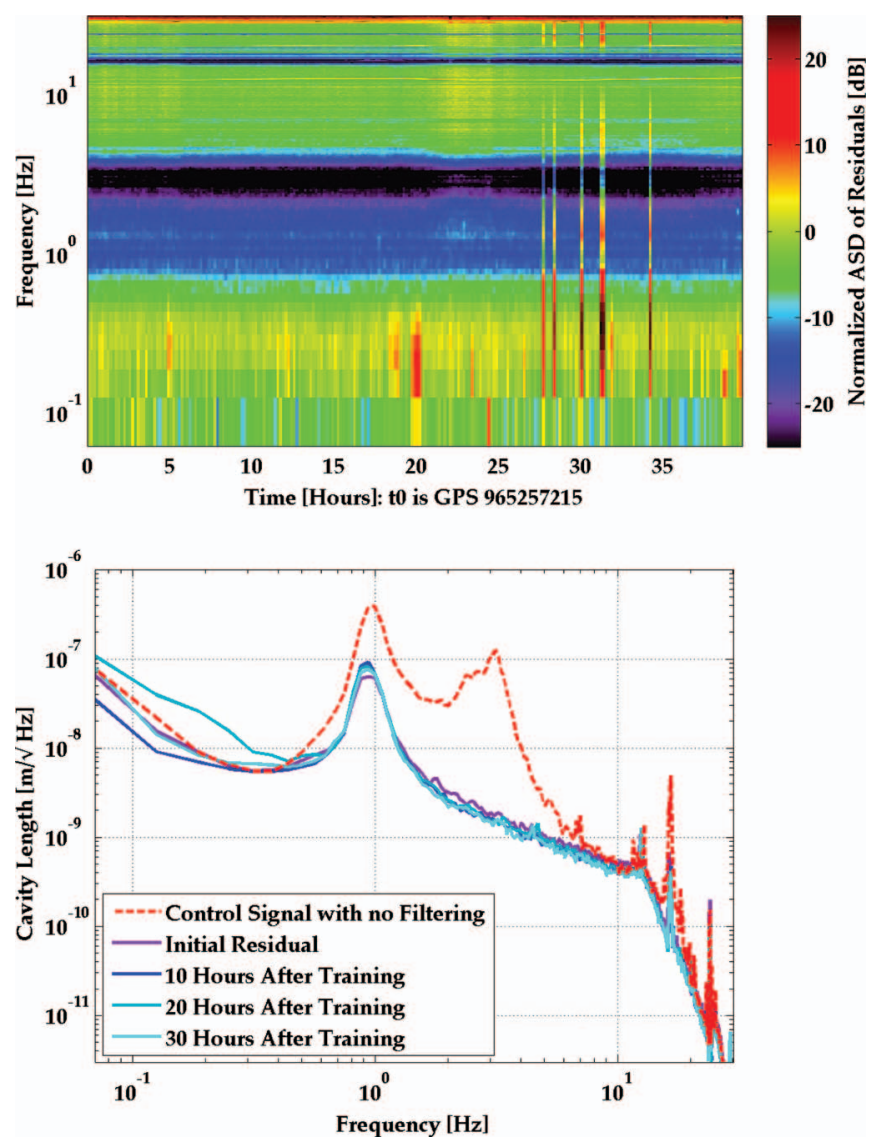

FIG. 2. (Color online) Result of offline seismic Wiener filtering on suspended triangular cavity. (a) Spectrogram showing the efficacy of a Wiener filter applied offline over a several hours period. Noticeably different traces between $\sim 28 \mathrm{~h}$ and $\sim 34 \mathrm{~h}$ are the result of non-stationary anthropogenic noise, not a decay of the filter's efficacy. (b) Amplitude spectral density of the control signal. Dotted trace is without subtraction, solid traces are 0,10 , 20 and 30 hours after filter was trained.

to 10 min segments of data for one day, using a $31 \mathrm{~s} \mathrm{long,}$ 2000 tap filter with a sample rate of $64 \mathrm{~Hz}$. In Figure 2(b), we select a few typical traces to illustrate the capabilities of the filter, while in Figure 2(a) we show the full results as a spectrogram, whitened by normalizing to the spectra during the time, the filter was being trained. We see large amounts of noise reduction both at the broad stack peak at $\sim 3 \mathrm{~Hz}$ and around the $16 \mathrm{~Hz}$ vertical mode of the mirror pendula.

We also include the noise contributions of our seismometers in Figure 3 to demonstrate how close we are able to get to the fundamental limit of Wiener filtering. Since the Wiener filter accepts, as inputs, the signals from the witness sensors (which have true ground motion information plus self-noise of the instruments and noise in the readout electronics), all of these noise contributions are filtered and added back into our data stream, limiting our ability to suppress ground motion below these levels. In Figure 3, we show that the differential ground motion over the length of the cavity is not much larger than the instrument noise of the seismometers. In other words, the ground noise over length of the cavity is strongly correlated below $\sim 1 \mathrm{~Hz}$ and so the differential motion is much smaller than the motion of any individual sensor. Currently our measurement of the differential ground motion is limited by the apparent instrument noise of the seismome- 


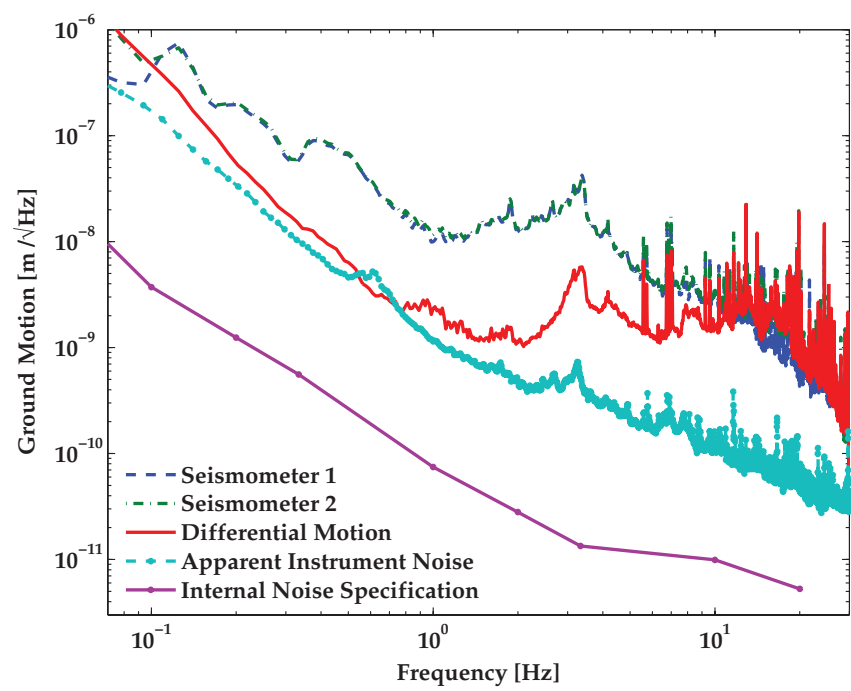

FIG. 3. (Color online) Shown are the spectra of the individual seismometers (dashed and dash-dot), the manufacturer's spec for the seismometers' internal noise (solid-circle), and the differential ground motion along the $13.5 \mathrm{~m}$ length of the cavity (solid). We also show the differential noise of the seismometers with the seismometers collocated in a stiff seismic vault (dashcircle); in principle, this is a measurement of the actual seismometer noise floor. It is unknown what uncorrelated noise is present in our sensors which makes the teal trace so much larger than the specification.

ters, represented by the teal trace in Figure 3. The apparent instrument noise is significantly higher than the specification, which indicates that there is some unknown noise which is uncorrelated between two seismometers, even when they are placed very close together. We will use lower noise sensors and readout electronics and better thermal/acoustic isolation of the seismometers in order to get better performance on such short baselines.

The limit to the performance of the feed-forward subtraction seems to be a combination of low frequency noise in the seismometers and the feedthrough of noise from the auxiliary controls systems of the cavity (e.g., angular controls, pendulum damping servos, etc.).

\section{APPLYING WIENER FILTERING TO A 4 km LIGO INTERFEROMETER}

One of the LIGO sites in Livingston, Lousisiana has had a hydraulic external pre-isolator (HEPI) actuation system installed since 2004 (the other LIGO site in Hanford, Washington will receive a HEPI system as part of the advanced LIGO upgrade). ${ }^{11}$ This HEPI system is designed to actuate on the seismic isolation stacks which support the suspended LIGO optics to actively reduce seismic noise. Initial implementation of the HEPI actuators only included local seismic isolation between 0.1 and $5 \mathrm{~Hz}$ to reduce anthropogenic noise, tidal effects, and the microseism. ${ }^{12}$

To estimate how the global Wiener filtering technique should scale up to a full size interferometer, we analyzed data from the 5th LIGO Science Run. ${ }^{1}$ While this analysis was done as offline post-processing, results from later tests executed on the LIGO interferometers using the HEPI actuators during the 6th LIGO Science Run will be available in a future paper. $^{13}$

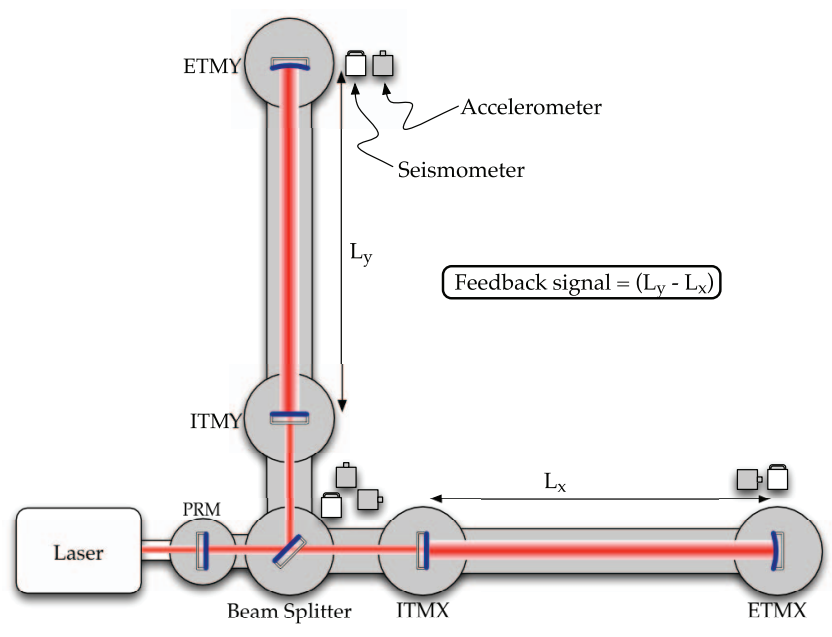

FIG. 4. (Color online) Schematic layout of seismometers relative to interferometer mirrors.

Instead of a single cavity, in this case we explored the subtraction of seismic noise from the differential arm length feedback signal (which is an accurate measure of the low frequency ground noise). The sensors are placed close to the ends of the interferometer arms and at the beam splitter as shown in Figure 4.

Figure 5 shows the resulting subtraction efficacy for a static filter. The variation in the $0.1-0.3 \mathrm{~Hz}$ band comes from variation in the ambient level of the double frequency microseismic peak. ${ }^{14}$ The structure in the $1-15 \mathrm{~Hz}$ band is the usual increase in anthropogenic noise during the workday.

Even though some excess noise is added in the dips around $3-5 \mathrm{~Hz}$ and $7 \mathrm{~Hz}$, the filter reduces the main contributors to the rms of the control signal, and the reduction is remarkably stable over the 30 days timespan. This static filter does inject an unacceptable amount of noise above $20 \mathrm{~Hz}$, which we will eliminatee in the future by using more aggressive pre-weighting to disallow such noise amplification before calculating the Wiener filter.

Figure 6 shows the subtraction if we use an acausal filter, retraining it every $10 \mathrm{~min}$, for the same 30 days data set. This filter performs much better than the static version. While we cannot apply an acausal filter in realtime, we can utilize causal adaptive filters to achieve nearly the same effect as long as the seismic environment does not change appreciably on time scales less than $10 \mathrm{~min}$.

Residuals for both Figures 5 and 6 were calculated using $46 \mathrm{~s}$ long Wiener filters of 3000 taps at a sample rate of $64 \mathrm{~Hz}$.

\section{ONLINE ADAPTIVE FILTERING AT THE $40 \mathrm{~m}$ INTERFEROMETER}

In case the transfer functions between the sensors and the target are changing with time, it would be useful to use a filter whose coefficients change with time. Such an adaptive filter could also take into account changes in the "actuator." The most simple and common implementation of an adaptive filter is the least mean squares (LMS) algorithm. ${ }^{15}$

The online adaptive filtering (OAF) algorithm implemented at the $40 \mathrm{~m}$ lab is the filtered-x least mean squares (FxLMS) algorithm. ${ }^{16}$ It is based primarily on the canonical 

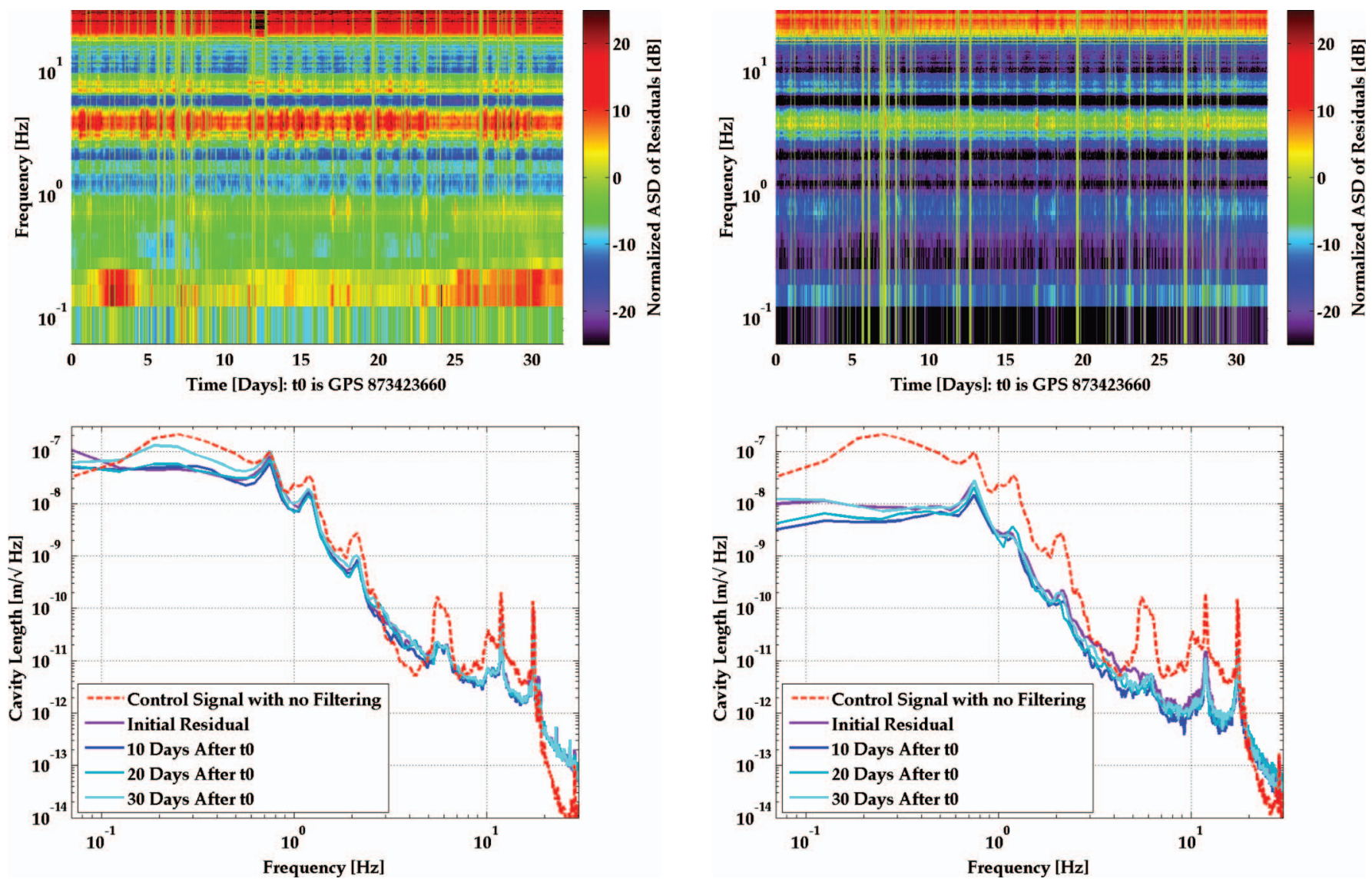

FIG. 5. (Color online) Result of offline simulated seismic Wiener filtering on the $4 \mathrm{~km}$ LIGO Hanford interferometer. (a) Traces are amplitude spectra normalized to the unfiltered control signal (dotted trace in $b$ ), which is at a time during the filter's training. Filter was trained on $6 \mathrm{~h}$ of data, then applied in 10 min segments. Vertical stripes indicate times when the interferometer was not operational. Seismic subtraction is fairly constant on a one month time scale, although it is not particularly effective for times when seismic noise is significantly different from the training time. (b) Selected individual spectra from (a) above. Dotted trace is before subtraction, solid traces are 0 , 10, 20 and 30 days after the filter was trained.

LMS algorithm; a steepest descent optimization of a defined error function. Just as in the static Wiener filtering in Sec. II, we minimize the rms of the difference between the filtered output and the original feedback signal. The LMS algorithm described in Eq. (4) takes "steps" in the direction of the steepest gradient until it arrives at a local minima.

$$
w(n+1)=w(n) \times[1-\tau]+\mu \times e(n) \times x(n) .
$$

Here the next iteration's FIR coefficients depend on the current coefficients $(w)$, the current witness signal $(x)$, the current error signal (difference between the target and filtered signal, $e$ ), and the adaptation rate $(\mu)$. One of the largest challenges with the adaptive filtering algorithm is that the success of the algorithm is fairly sensitive to the choice of $\mu$. To improve stability against transients, we modify the usual FxLMS algorithm to include a decay constant $\tau$.

The FxLMS algorithm acknowledges that there exist phase delays in the path of the target signal which cannot be approximated by the LMS method alone. ${ }^{17}$ To account for these phase delays, we filter the incoming witness signals with filters identical to those in the target signal path. Once we have

FIG. 6. (Color online) Result of offline simulated seismic Wiener filtering on the $4 \mathrm{~km}$ LIGO Hanford interferometer, using an acausal filter on the same 30 days data set. (a) Traces are amplitude spectra normalized to the unfiltered control signal (dotted trace in b). A filter is trained on, and then applied to, 10 min segments of data. Seismic noise is more effectively suppressed using this constantly updated filter, implying that the transfer function is changing on a relatively short time scale, and that it is advantageous to update the filter more often than once per month. (b) Selected individual spectra from (a) above. Dotted trace is before subtraction, solid traces are 0, 10, 20 and 30 days after beginning.

matched the delays in the two different paths, we implement the regular LMS optimization to find the coefficients we will use in our FIR filter. The FxLMS algorithm used is sketched in Figure 7.

We apply the OAF system to the same triangular cavity as in Sec. III. Once again, we use the cavity length

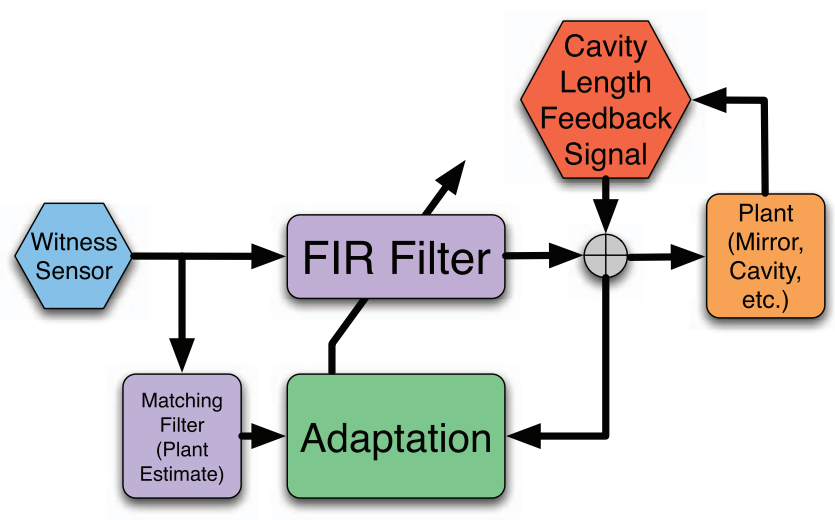

FIG. 7. (Color online) Block diagram of the FxLMS algorithm used. 


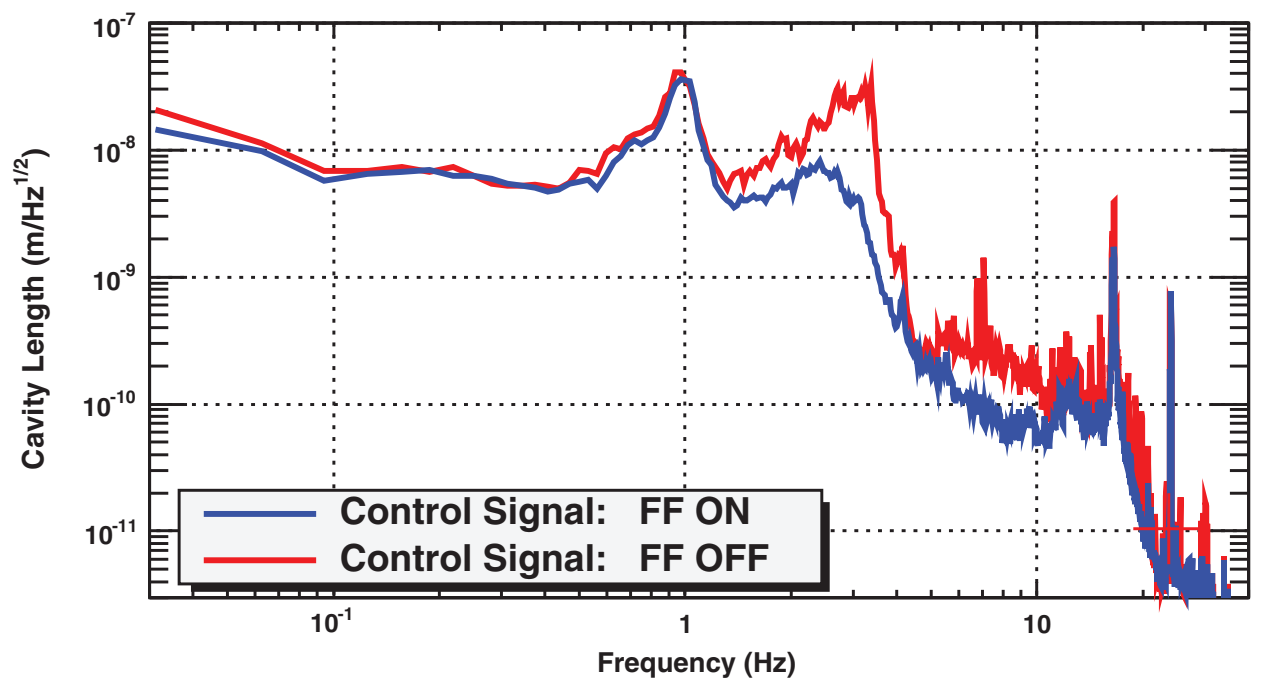

FIG. 8. (Color online) Online adaptive filter performance: the spectral density of the cavity length fluctuations are shown with the feed-forward on (lower trace) and off (upper trace).

feedback signal as our targeted signal to minimize, and a similar layout of independent witness sensors as shown in Figure 1. Unlike Secs. III and IV which were simulations using previously collected data, here we are actuating on the cavity in realtime. Figure 8 shows results using a $125 \mathrm{~s}$ long, 2000 tap filter with $\mu=0.01$ and $\tau=10^{-6}$ at a $16 \mathrm{~Hz}$ sample rate. The on/off traces in the adaptive case are similar to estimates made in the static Wiener filtering case (Figure 2). Given enough time to adapt, the OAF converges towards the optimal filter, but, so far, not completely. Since the adaptive system was tested using one Güralp seismometer and one Ranger SS-1 seismometer, the subtraction is not as pronounced as if we had used two Güralps, or other more sensitive broadband seismometers. In the next iteration of this setup, we will explore the variation in the cancellation performance as a function of sensor placement.

\section{CONCLUSIONS}

We have demonstrated the use of Wiener filter based feed-forward seismic noise reduction on a suspended interferometer. We have also implemented a stable, adaptive feedforward system which has a performance approaching that of the optimal Wiener estimate. These techniques can be simply implemented in any general laboratory requiring vibration isolation using relatively low cost accelerometers and commodity computers and digital signal processing software (e.g., LabVIEW). These "optimal" feed-forward schemes operate without having to know a priori the transfer function between the disturbance and the primary experiment; they can easily be reconfigured to adapt to new experimental setups. Similar Wiener filter and LMS based techniques have been utilized in other experiments, both offline and online, for example, isolating two-mirror Fabry-Perot cavities from ground motion, ${ }^{18}$ reducing acoustic noise in oceanography settings, ${ }^{19}$ and in signal processing to decorrelate degenerate witness channels. $^{20}$

In the near future, we will work to use this scheme to reduce the noise in multiple degrees of freedom of the full interferometer. It is clear that this technique can also be applied to remove other sources of environmental noise (e.g., acoustic, magnetic, electronic, etc.). For each noise source in the gravitational wave band, we will inject software gravitational wave signals into the data stream and confirm that they are not distorted. There will certainly be new challenges associated with each type of noise, but this seems promising as a method which can be employed to reduce the influence of environmental noise in a wide variety of experimental setups.

\section{ACKNOWLEDGMENTS}

We gratefully acknowledge illuminating discussions with Joe Giaime, Alan Weinstein, Rob Ward, and Jan Harms. We also thank the National Science Foundation (NSF) for support under grant PHY-0555406. J. Driggers also acknowledges the support of an NSF Graduate Research Fellowship. K. Pepper acknowledges the support of the LIGO NSF REU program. LIGO was constructed by the California Institute of Technology and Massachusetts Institute of Technology with funding from the National Science Foundation (NSF) and operates under cooperative agreement PHY-0107417. This article has LIGO Document Number P0900071.

${ }^{1}$ B. P. Abbott, R. Abbott, R. Adhikari, P. Ajith, B. Allen, G. Allen, R. S. Amin, S. B. Anderson, W. G. Anderson, M. A. Arain, M. Araya, H. Armandula, P. Armor, Y. Aso, S. Aston, P. Aufmuth, C. Aulbert, S. Babak, P. Baker, S. Ballmer, C. Barker, D. Barker, B. Barr, P. Barriga, L. Barsotti, M. A. Barton, I. Bartos, R. Bassiri, M. Bastarrika, B. Behnke, M. Benacquista, J. Betzwieser, P. T. Beyersdorf, I. A. Bilenko, G. Billingsley, R. Biswas, E. Black, J. K. Blackburn, L. Blackburn, D. Blair, B. Bland, T. P. Bodiya, L. Bogue, R. Bork, V. Boschi, S. Bose, P. R. Brady, V. B. Braginsky, J. E. Brau, D. O. Bridges, M. Brinkmann, A. F. Brooks, D. A. Brown, A. Brummit, G. Brunet, A. Bullington, A. Buonanno, O. Burmeister, R. L. Byer, L. Cadonati, J. B. Camp, J. Cannizzo, K. C. Cannon, J. Cao, L. Cardenas, S. Caride, G. Castaldi, S. Caudill, M. Cavaglia, C. Cepeda, T. Chalermsongsak, E. Chalkley, P. Charlton, S. Chatterji, S. Chelkowski, Y. Chen, N. Christensen, C. T. Y. Chung, D. Clark, J. Clark, J. H. Clayton, T. Cokelaer, C. N. Colacino, R. Conte, D. Cook, T. R. C. Corbitt, N. Cornish, D. Coward, D. C. Coyne, J. D. E. Creighton, T. D. Creighton, A. M. Cruise, R. M. Culter, A. Cumming, L. Cunningham, S. L. Danilishin, K. Danzmann, B. Daudert, G. Davies, 
E. J. Daw, D. DeBra, J. Degallaix, V. Dergachev, S. Desai, R. DeSalvo, S. Dhurandhar, M. Diaz, A. Dietz, F. Donovan, K. L. Dooley, E. E. Doomes, R. W. P. Drever, J. Dueck, I. Duke, J.-C. Dumas, J. G. Dwyer, C. Echols, M. Edgar, A. Effler, P. Ehrens, E. Espinoza, T. Etzel, M. Evans, T. Evans, S. Fairhurst, Y. Faltas, Y. Fan, D. Fazi, H. Fehrmenn, L. S. Finn, K. Flasch, S. Foley, C. Forrest, N. Fotopoulos, A. Franzen, M. Frede, M. Frei, Z. Frei, A. Freise, R. Frey, T. Fricke, P. Fritschel, V. V. Frolov, M. Fyffe, V. Galdi, J. A. Garofoli, I. Gholami, J. A. Giaime, S. Giampanis, K. D. Giardina, K. Goda, E. Goetz, L. M. Goggin, G. Gonzalez, M. L. Gorodetsky, S. Gossler, R. Gouaty, A. Grant, S. Gras, C. Gray, M. Gray, R. J. S. Greenhalgh, A. M. Gretarsson, F. Grimaldi, R. Grosso, H. Grote, S. Grunewald, M. Guenther, E. K. Gustafson, R. Gustafson, B. Hage, J. M. Hallam, D. Hammer, G. D. Hammond, C. Hanna, J. Hanson, J. Harms, G. M. Harry, I. W. Harry, E. D. Harstad, K. Haughian, K. Hayama, J. Heefner, I. S. Heng, A. Heptonstall, M. Hewitson, S. Hild, E. Hirose, D. Hoak, K. A. Hodge, K. Holt, D. J. Hosken, J. Hough, D. Hoyland, B. Hughey, S. H. Huttner, D. R. Ingram, T. Isogai, M. Ito, A. Ivanov, B. Johnson, W. W. Johnson, D. I. Jones, G. Jones, R. Jones, L. Ju, P. Kalmus, V. Kalogera, S. Kandhasamy, J. Kanner, D. Kasprzyk, E. Katsavounidis, K. Kawabe, S. Kawamura, F. Kawazoe, W. Kells, D. G. Keppel, A. Khalaidovski, F. Y. Khalili, R. Khan, E. Khazanov, P. King, J. S. Kissel, S. Klimenko, K. Kokeyama, V. Kondrashov, R. Kopparapu, S. Koranda, D. Kozak, B. Krishnan, R. Kumar, P. Kwee, P. K. Lam, M. Landry, B. Lantz, A. Lazzarini, H. Lei, M. Lei, N. Leindecker, I. Leonor, C. Li, H. Lin, P. E. Lindquist, T. B. Littenberg, N. A. Lockerbie, D. Lodhia, M. Longo, M. Lormand, P. Lu, M. Lubinski, A. Lucianetti, H. Lueck, B. Machenschalk, M. MacInnis, M. Mageswaran, K. Mailand, I. Mandel, V. Mandic, S. Marka, Z. Marka, A. Markosyan, J. Markowitz, E. Maros, I. W. Martin, R. M. Martin, J. N. Marx, K. Mason, F. Matichard, L. Matone, R. A. Matzner, N. Mavalvala, R. McCarthy, D. E. McClelland, S. C. McGuire, M. McHugh, G. McIntyre, D. J.A. McKechan, K. McKenzie, M. Mehmet, A. Melatos, A. C. Melissinos, D. F. Menendez, G. Mendell, R. A. Mercer, S. Meshkov, C. Messenger, M. S. Meyer, J. Miller, J. Minelli, Y. Mino, V. P. Mitrofanov, G. Mitselmakher, R. Mittleman, O. Miyakawa, B. Moe, S. D. Mohanty, S. R.P. Mohapatra, G. Moreno, T. Morioka, K. Mors, K. Mossavi, C. MowLowry, G. Mueller, H. Mueller-Ebhardt, D. Muhammad, S. Mukherjee, H. Mukhopadhyay, A. Mullavey, J. Munch, P. G. Murray, E. Myers, J. Myers, T. Nash, J. Nelson, G. Newton, A. Nishizawa, K. Numata, J. O’Dell, B. O'Reilly, R. O'Shaughnessy, E. Ochsner, G. H. Ogin, D. J. Ottaway, R. S. Ottens, H. Overmier, B. J. Owen, Y. Pan, C. Pankow, M. A. Papa, V. Parameshwaraiah, P. Patel, M. Pedraza, S. Penn, A. Perraca, V. Pierro, I. M. Pinto, M. Pitkin, H. J. Pletsch, M. V. Plissi, F. Postiglione, M. Principe, R. Prix, L. Prokhorov, O. Punken, V. Quetschke, F. J. Raab, D. S. Rabeling, H. Radkins, P. Raffai, Z. Raics, N. Rainer, M. Rakhmanov, V. Raymond, C. M. Reed, T. Reed, H. Rehbein, S. Reid, D. H. Reitze, R. Riesen, K. Riles, B. Rivera, P. Roberts, N. A. Robertson, C. Robinson, E. L. Robinson, S. Roddy, C. Roever, J. Rollins, J. D. Romano, J. H. Romie, S. Rowan, A. Ruediger, P. Russell, K. Ryan, S. Sakata, L. Sancho de la Jordana, V. Sandberg, V. Sannibale, L. Santamaria, S. Saraf, P. Sarin, B. S. Sathyaprakash, S. Sato, M. Satterthwaite, P. R. Saulson, R. Savage, P. Savov, M. Scanlan, R. Schilling, R. Schnabel, R. Schofield, B. Schulz, B. F. Schutz, P. Schwinberg, J. Scott, S. M. Scott, A. C. Searle, B. Sears, F. Seifert, D. Sellers, A. S. Sengupta, A. Sergeev, B. Shapiro, P. Shawhan, D. H. Shoemaker, A. Sibley, X. Siemens, D. Sigg, S. Sinha, A. M. Sintes, B. J. J. Slagmolen, J. Slutsky, J. R. Smith, M. R. Smith, N. D. Smith, K. Somiya, B. Sorazu, A. Stein, L. C. Stein, S. Steplewski, A. Stochino, R. Stone, K. A. Strain, S. Strigin, A. Stroeer, A. L. Stuver, T. Z. Summerscales, K-X. Sun, M. Sung, P. J. Sutton, G. P. Szokoly, D. Talukder, L. Tang, D. B. Tanner, S. P. Tarabrin, J. R. Taylor, R. Taylor, J. Thacker, K. A. Thorne, A. Thuering, K. V. Tokmakov, C. Torres, C. Torrie, G. Traylor, M. Trias, D. Ugolini, J. Ulmen, K. Urbanek, H. Vahlbruch, M. Vallisneri, C. Van Den Broeck, M. V. van der Sluys, A. A. van Veggel, S. Vass, R. Vaulin, A. Vecchio, J. Veitch, P. Veitch, C. Veltkamp, A. Villar, C. Vorvick, S. P. Vyachanin, S. J. Waldman, L. Wallace, R. L. Ward, A. Wei- dner, M. Weinert, A. J. Weinstein, R. Weiss, L. Wen, S. Wen, K. Wette, J. T. Whelan, S. E. Whitcomb, B. F. Whiting, C. Wilkinson, P. A. Willems, H. R. Williams, L. Williams, B. Willke, I. Wilmut, L. Winkelmann, W. Winkler, C. C. Wipf, A. G. Wiseman, G. Woan, R. Wooley, J. Worden, W. Wu, I. Yakushin, H. Yamamoto, Z. Yan, S. Yoshida, M. Zanolin, J. Zhang, L. Zhang, C. Zhao, N. Zotov, M. E. Zucker, H. zur Muehlen, and J. Zweizig, Rep. Prog. Phys. 72, 076901 (2009).

${ }^{2}$ P. R. Saulson, Phys. Rev. D 30, 732 (1984).

${ }^{3}$ S. A. Hughes and K. S. Thorne, Phys. Rev. D 58, 122002 (1998).

${ }^{4}$ M. Beccaria, M. Bernardini, S. Braccini, C. Bradaschia, A. Bozzi, C. Casciano, G. Cella, A. Ciampa, E. Cuoco, G. Curci, E. D'Ambrosio, V. Dattilo, G. De Carolis, R. De Salvo, A. Di Virgilio, A. Delapierre, D. Enard, A. Errico, G. Feng, I. Ferrante, F. Fidecaro, F. Frasconi, A. Gaddi, A. Gennai, G. Gennaro, A. Giazotto, P. La Penna, G. Losurdo, M. Maggiore, S. Mancini, F. Palla, H. B. Pan, F. Paoletti, A. Pasqualetti, R. Passaquieti, D. Passuillo, R. Poggiani, P. Popolizio, F. Raffaelli, S. Rapisarda, A. Vicere, and Z. Zhang, Class. Quantum Grav. 15, 3339 (1998).

${ }^{5}$ S. Haykin, Adaptive Filter Theory, 4th ed. (Prentice Hall, Englewood Cliffs, NJ, 2002).

${ }^{6}$ J. Durbin, Rev. Inst. Int. Stat. 28, 233 (1960).

${ }^{7}$ Y. Huang, J. Benesty, and J. Chen, Acoustic MIMO Signal Processing, Signals and Communication Technology (Springer, New York, 2006).

${ }^{8} \mathrm{R}$. Ward, "Length sensing and control of a prototype advanced interferometric gravitational wave detector," Ph.D. dissertation (California Institute of Technology, 2010).

${ }^{9} \mathrm{~J}$. A. Giaime, "Studies of laser interferometer design and a vibration isolation system for interferometric gravitational wave detectors," Ph.D. dissertation (Massachusetts Institute of Technology, 1995).

${ }^{10}$ J. A. Giaime, P. Saha, D. Shoemaker, and L. Sievers, Rev. Sci. Instrum. 67, 208 (1996).

${ }^{11}$ N. A. Robertson, B. Abbott, R. Abbott, R. Adhikari, G. Allen, H. Armandula, S. Aston, A. Baglino, M. Barton, B. Bland, R. Bork, J. Bogenstahl, G. Cagnoli, C. Campbell, C. A. Cantley, K. Carter, D. Cook, D. Coyne, D. Crooks, E. Daw, D. DbBra, E. Elliffe, J. Faludi, P. Fritschel, A. Ganguli, J. Giaime, S. Gossler, A. Grant, J. Greenhalgh, M. Hammond, J. Hanson, C. Hardham, G. Harry, A. Heptonstall, J. Heefner, J. Hough, D. Hoyland, W. Hua, L. Jones, R. Jones, J. Kern, J. LaCour, B. Lantz, K. Lilienkamp, N. Lockerbie, H. Lueck, M. MacInnis, K. Mailand, K. Mason, R. Mittleman, S. Nayfeh, J. Nochol, D. J. Ottaway, H. Overmier, M. Perreur-Lloyd, J. Phinney, M. Plissi, W. Rankin, D. Robertson, J. Romie, S. Rowan, R. Scheffler, D. H. Shoemaker, P. Sarin, P. Sneddon, C. Speake, O. Spjeld, G. Stapfer, K. A. Strain, C. Torrie, G. Traylor, J. van Niekerk, A. Vecchio, S. Wen, P. Willems, I. Wilmut, H. Ward, M. Zucker, and L. Zuo, Proc. SPIE 5500, 81 (2004).

${ }^{12} \mathrm{~S}$. Wen, "Improved seismic isolation for the laser interferometer gravitational wave observatory with hydraulic external preisolator system," $\mathrm{Ph} . \mathrm{D}$. dissertation (Louisiana State University, 2009).

${ }^{13}$ R. deRosa, J. Driggers, D. Atkinson, H. Miao, V. Frolov, M. Landry, and R. Adhikari, "Global feed-forward vibration isolation in a km scale interferometer," (unpublished).

${ }^{14}$ R. A. Haubrich and K. McCamy, Rev. Geophys. 7, 539, doi:10.1029/RG007i003p00539 (1969).

${ }^{15}$ A. H. Sayed, Fundamentals of Adaptive Filtering (IEEE, New York, 2003).

${ }^{16}$ B. Widrow and S. D. Stearns, Adaptive Signal Processing (Prentice Hall, Englewood Cliffs, NJ, 1985).

${ }^{17}$ National Instruments, "Filtered-X LMS Algorithms," LabVIEW Adaptive Filter Toolkit 1.0 Help, June 2008 (http://zone.ni.com/ reference/en-XX/help/372357A-01/lvaftconcepts/aft_filteredx/).

${ }^{18}$ M. J. Thorpe, D. R. Leibrandt, T. M. Fortier, and T. Rosenband, Opt. Express 18, 18744 (2010).

${ }^{19} \mathrm{C}$. W. Therrien, K. L. Frack, Jr., and N. R. Fontes, in IEEE International Conference on Acoustics, Speech and Signal Processing, Munich, Germany (IEEE Computer Society Press, Los Alamitos, California), pp. 543-546.

${ }^{20}$ Y. Tu and C. R. Fuller, J. Sound Vib. 233, 761 (2000). 